PROCEEDINGS OF THE

AMERICAN MATHEMATICAL SOCIETY

Volume 129, Number 12, Pages 3657-3664

S 0002-9939(01)05994-9

Article electronically published on May 3, 2001

\title{
ON SUPPORT POINTS OF UNIVALENT FUNCTIONS AND A DISPROOF OF A CONJECTURE OF BOMBIERI
}

\author{
RICHARD GREINER AND OLIVER ROTH
}

(Communicated by Albert Baernstein II)

\begin{abstract}
We consider the linear functional $\operatorname{Re}\left(a_{3}+\lambda a_{2}\right)$ for $\lambda \in i \mathbb{R}$ on the set of normalized univalent functions in the unit disk and use the result to disprove a conjecture of Bombieri.
\end{abstract}

\section{INTRODUCTION}

Let $\mathcal{S}$ be the class of functions $f(z)=z+a_{2} z^{2}+a_{3} z^{3}+\cdots$ analytic and univalent in the unit disk $\mathbb{D}:=\{z \in \mathbb{C}:|z|<1\}$. We consider for a fixed constant $\lambda \in \mathbb{C}$ the linear functional

$$
L_{\lambda}(f)=L_{\lambda}\left(a_{2}, a_{3}\right):=\operatorname{Re}\left(a_{3}+\lambda a_{2}\right), \quad f \in \mathcal{S} .
$$

Every function $F \in \mathcal{S}$ maximizing $L_{\lambda}$ over $\mathcal{S}$ is called a support point for $L_{\lambda}$.

The coefficient functional $L_{\lambda}$ has been studied by Brown [Bro81] who obtained a complete picture of the support points for $L_{\lambda}$ for all $\lambda \in \mathbb{C}$ apart from the case $\lambda= \pm i|\lambda|, 6 \leq|\lambda|<8$. In a completely different manner the functional $L_{\lambda}$ was investigated by Tammi and Kortram in [KT80 and by Tammi in Tam82. However, the reasoning in KT80] and Tam82 in the most difficult case $\lambda \in i \mathbb{R},|\lambda|<8$, is not complete as it was pointed out for instance by Leung, Leu85, p. 9. The problem is to show that a certain system of non-linear equations (cf. (54) in [Tam82], p. 87) has a unique solution; see also the remarks in Haa83, p. 65.

It is the purpose of this note to fill in this gap and to give a rigorous proof of the following result stated by Tammi Tam82, p. 90.

Theorem 1.1. Let $\lambda \in i \mathbb{R} \backslash\{0\}$ and let $F \in \mathcal{S}$ be a support point for the functional (1.1) over $\mathcal{S}$ :

(a) If $|\lambda| \geq 4 e /(e-1)=6.3279 \ldots$ and $\lambda=i|\lambda|$ (resp. $\lambda=-i|\lambda|)$, then $F(z)=$ $i K(-i z)$ (resp. $F(z)=-i K(i z))$ where $K(z)=z /(1-z)^{2}$ is the Koebe function.

(b) If $0<|\lambda|<4 e /(e-1)$, then $F$ is not a rotation of the Koebe function.

Received by the editors December 28, 1999 and, in revised form, May 1, 2000.

1991 Mathematics Subject Classification. Primary 30C70, 30C50; Secondary 30C35.

Key words and phrases. Univalent functions, support point, linear functional, fractional linear functional, Schiffer variation.

This paper was completed while the second author was visiting the University of Michigan supported by a Feodor Lynen fellowship of the Alexander von Humboldt foundation. He thanks the faculty and staff for their hospitality. 
The proof of Theorem 1.1 will be given in Section 2 .

The information on the linear functional $L_{\lambda}$ provided by Theorem[1.1 can be used to solve a linear fractional extremal problem on $\mathcal{S}$ and leads to a precise statement about the coeffcient body $V_{3}:=\left\{\left(a_{2}, a_{3}\right)^{T}: f \in \mathcal{S}\right\}$ near the "Koebe-point":

Corollary 1.2. We have

$$
\min _{\left(a_{2}, a_{3}\right) \in V_{3}} \frac{2-\operatorname{Re} a_{2}}{3-\operatorname{Re} a_{3}}=\liminf _{a_{2} \rightarrow 2} \frac{2-\operatorname{Re} a_{2}}{3-\operatorname{Re} a_{3}}=\frac{1}{4} \frac{e-1}{e}=0.15803 \ldots,
$$

where the liminf are taken over all functions of $\mathcal{S}$.

Proof. Using the rotation invariance of $\mathcal{S}$ it is easy to see that

$$
\min _{f \in \mathcal{S}} \operatorname{Re}\left(a_{3}-\lambda a_{2}\right)=-\max _{f \in \mathcal{S}} \operatorname{Re}\left(a_{3}+i \lambda a_{2}\right), \quad \lambda \in \mathbb{C} .
$$

Therefore, Theorem 1.1 (a) implies

$$
\frac{3-\operatorname{Re} a_{3}}{2-\operatorname{Re} a_{2}} \leq 4 \frac{e}{e-1}
$$

for all $f \in \mathcal{S}, f \neq K$, i.e.,

$$
\min _{\left(a_{2}, a_{3}\right) \in V_{3}} \frac{2-\operatorname{Re} a_{2}}{3-\operatorname{Re} a_{3}} \geq \frac{1}{4} \frac{e-1}{e} .
$$

Let $0<\lambda_{n}<4 e /(e-1)$ such that $\lim _{n \rightarrow \infty} \lambda_{n}=4 e /(e-1)$. In view of (1.3) and Theorem 1.1 (b) there are functions $F_{n}(z)=z+a_{2}^{(n)} z^{2}+a_{3}^{(n)} z^{3}+\cdots \in \mathcal{S} \backslash\{K\}$ such that $F_{n} \rightarrow K$ locally uniformly in $\mathbb{D}$ and

$$
\min _{f \in \mathcal{S}} \operatorname{Re}\left(a_{3}-\lambda_{n} a_{2}\right)=\operatorname{Re}\left(a_{3}^{(n)}-\lambda_{n} a_{2}^{(n)}\right)<3-2 \lambda_{n}
$$

In particular, $3-\operatorname{Re} a_{3}^{(n)} \geq \lambda_{n}\left(2-\operatorname{Re} a_{2}^{(n)}\right)$, i.e.,

$$
\liminf _{a_{2} \rightarrow 2} \frac{2-\operatorname{Re} a_{2}}{3-\operatorname{Re} a_{3}} \leq \lim _{n \rightarrow \infty} \frac{2-\operatorname{Re} a_{2}^{(n)}}{3-\operatorname{Re} a_{3}^{(n)}} \leq \lim _{n \rightarrow \infty} \frac{1}{\lambda_{n}}=\frac{1}{4} \frac{e-1}{e} .
$$

The assertion follows now from inequalities (1.4) and (1.5).

In particular, Corollary 1.2 disproves a conjecture of Bombieri [Bom67], p. 51 (see also BH85, [BH87]), which asserts that

$$
\liminf _{a_{m} \rightarrow m} \frac{n-\operatorname{Re} a_{n}}{m-\operatorname{Re} a_{m}}=\min _{\theta \in \mathbb{R}} \frac{n-\frac{\sin (n \theta)}{\sin \theta}}{m-\frac{\sin (m \theta)}{\sin \theta}} \quad \text { for all } m, n \geq 2,
$$

in the case $n=2$ and $m=3$, because for $n=2$ and $m=3$ the right-hand side of (1.6) equals $1 / 4$ which is strictly larger than the bound $(e-1) /(4 e)$ in Corollary 1.2

\section{Proof of Theorem 1.1}

The standard method of boundary variation ( $c f$. [SSp50], [Dur83]) shows that every support point $F(z)=z+A_{2} z^{2}+A_{3} z^{3}+\cdots$ of the functional $L_{\lambda}, \lambda \in \mathbb{C}$ fixed, is a solution of the Schiffer differential equation

$$
\left[\frac{z F^{\prime}(z)}{F(z)}\right]^{2} \frac{1+A F(z)}{F(z)^{2}}=\frac{1}{z^{2}}+\frac{A}{z}+B_{0}+\bar{A} z+z^{2}
$$


where

$$
B_{0}=2 A_{3}+\lambda A_{2}>0, \quad A=2 A_{2}+\lambda .
$$

In the sequel we will work only with conditions (2.1) and (2.2). It is therefore convenient to introduce the following terminology; $c f$. [Pfl88].

Definition 2.1. A function $f(z)=z+a_{2} z^{2}+\cdots \in \mathcal{S}$ is called A-admissible for $A \in \mathbb{C}$ if $f$ admits a piecewise analytic extension to $\overline{\mathbb{D}}$ such that

$$
z \mapsto\left[\frac{z f^{\prime}(z)}{f(z)}\right]^{2} \frac{1+A f(z)}{f(z)^{2}}
$$

is positive on $|z|=1$ except possibly for one or two points on $|z|=1$. If, in addition, $A=2 a_{2}+\lambda$, then $f$ is called a critical point for the functional $L_{\lambda}$.

Therefore, every support point $F(z)=z+A_{2} z^{2}+\cdots$ for $L_{\lambda}$ is $A$-admissible for $A=2 A_{2}+\lambda$, i.e., every support point for $L_{\lambda}$ is a critical point for $L_{\lambda}$.

Lemma 2.2. If $F(z)=z+A_{2} z^{2}+\cdots \in \mathcal{S}$ is a support point for $L_{\lambda}$, then $A=$ $2 A_{2}+\lambda \in \mathbb{C} \backslash(-4,4)$.

This follows from the fact that $A$-admissible functions for $A \in(-4,4)$ are twoslit mappings whereas support points in $\mathcal{S}$ are necessarily one-slit maps; $c f$. [SSp50] for details.

In the next lemma we shall consider $A$-admissible functions for $A \notin(-4,4)$. We parametrize $A$ in terms of $\rho \in(0,1]$ and $\phi \in(-\pi, \pi]$ by

$$
A=A(\rho, \phi):=\left(\rho+\frac{1}{\rho}\right) e^{i \phi}+2 e^{-i \phi} .
$$

(This is the parametrization used by Schaeffer and Spencer [SSp50], Chapter XIII.)

Lemma 2.3. If $A \in \mathbb{C} \backslash(-4,4)$, then there exists a uniquely determined $A$-admissible function $f_{A}(z)=z+a_{2}(A) z^{2}+\cdots \in \mathcal{S}$. Moreover $\mathbb{C} \backslash f_{A}(\mathbb{D})$ is an analytic Jordan arc extending to $\infty$ and

$$
2 a_{2}(A)=4 e^{-i \phi}-A \log \left(1+\rho^{2}+2 \rho e^{-2 i \phi}\right)+A \log \left(1-\rho^{2}\right)+\bar{A} \log \frac{1+\rho}{1-\rho} .
$$

Equation (2.4) is exactly formula (13.5.8) in [SSp50] for the part of the coefficient body $V_{3}$ which corresponds to one-slit mappings.

We now characterize the critical points of the functional $L_{\lambda}$ which are $A$-admissible for $A \notin(-4,4)$.

Lemma 2.4. If $f$ is a critical point of the functional $L_{\lambda}$ for $\lambda \in i \mathbb{R}$ which is $A$-admissible for $A=A(\rho, \phi) \in \mathbb{C} \backslash(-4,4)$, then $h(\rho, \phi)=0$ where

$$
\begin{aligned}
h(\rho, \phi):= & (\rho-1)^{2} \cos \phi-2(\rho+1)^{2} \log (1+\rho) \cos \phi \\
& +(\rho+1)^{2} \cos \phi \operatorname{Re}\left(\log \left(1+\rho^{2}+2 \rho e^{-2 i \phi}\right)\right) \\
& +(\rho-1)^{2} \sin \phi \operatorname{Im}\left(\log \left(1+\rho^{2}+2 \rho e^{-2 i \phi}\right)\right) .
\end{aligned}
$$


Moreover, $\lambda=i p(\rho, \phi)$, where

$$
\begin{aligned}
p(\rho, \phi)= & \operatorname{Im} A(\rho, \phi)-2 \operatorname{Im} a_{2}(A(\rho, \phi)) \\
= & \frac{(1+\rho)^{2}}{\rho} \cos \phi \operatorname{Im}\left(\log \left(1+2 \rho e^{-2 i \phi}+\rho^{2}\right)\right) \\
& +\frac{(1+\rho)^{2}-2(\rho-1)^{2} \log (1-\rho)}{\rho} \sin \phi \\
& +\frac{(\rho-1)^{2}}{\rho} \operatorname{Re}\left(\log \left(1+2 \rho e^{-2 i \phi}+\rho^{2}\right)\right) \sin \phi .
\end{aligned}
$$

Proof. We know from (2.2) that $A=2 a_{2}(A)+\lambda$ where $a_{2}(A)$ is given by (2.4). Taking the real part leads to $h(\rho, \phi)=0$; taking the imaginary part gives (2.6).

We now consider the equation $h(\rho, \phi)=0$. Since $h(\rho, \pi \pm \phi)=-h(\rho, \phi)$ we may restrict ourselves to the case $0<\rho \leq 1$ and $0 \leq \phi \leq \pi / 2$. For $\phi=0$ we have $h(\rho, \phi)=0$ if and only if $\rho=1$. In this case $\lambda=0$ and there are exactly two support points, namely $K(z)$ and $-K(-z)$.

Lemma 2.5. If $h(\rho, \phi)=0$ with $0<\rho \leq 1,0<\phi \leq \pi / 2$, then either:

(a) $\phi=\pi / 2$ and $p(\rho, \phi)=(1+\rho)^{2} / \rho \in[4, \infty)$, or

(b) $\phi<\pi / 2$. In this case $(\sqrt{e}-1) /(\sqrt{e}+1) \doteq 0.244919<\rho \leq 1$ and $\phi=\phi(\rho)$ is a continuously differentiable and strictly decreasing function of $(0,1]$ onto $[0, \pi / 2)$. Moreover the function $\rho \mapsto p(\rho, \phi(\rho))$ is continuously differentiable and strictly decreasing on $(0,1]$ and takes on its values in $[0,4 e /(e-1))$.
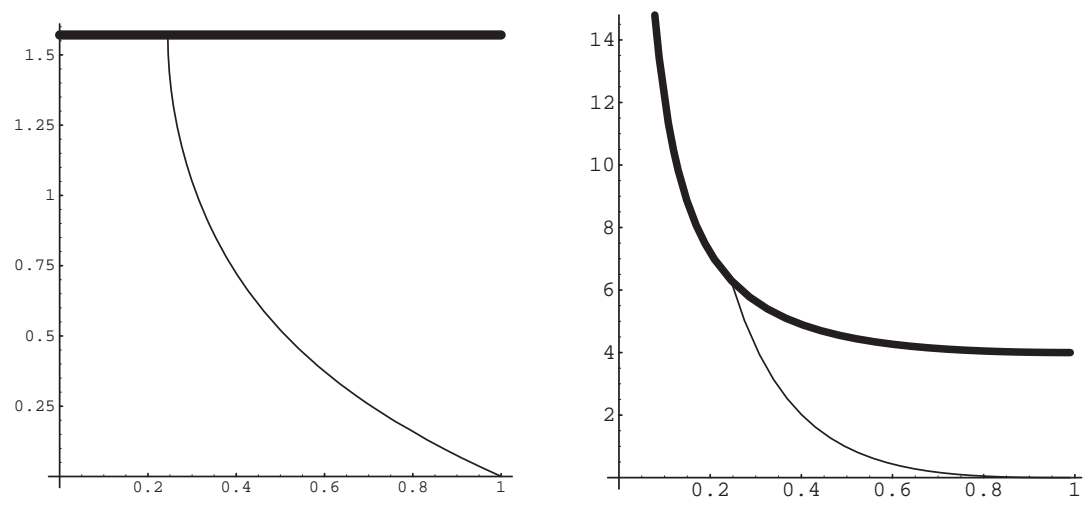

Figure 1. The locus of the zeros of $h(\rho, \phi)$ consisting of two curves in the $\rho$ - $\phi$-plane (on the left) and the values of $p$ as a function of $\rho$ along these curves (on the right). The thick parts correspond to the Koebe function $z /(1-i z)^{2}$.

Proof. (a) is obvious. To prove (b) we introduce the functions

$$
\begin{aligned}
g(v, x) & :=v+\frac{1}{2}[L(v, x)+2 \log v]+\frac{v s(x)}{4(1+x)} T(v, x), \\
q(v, x) & :=\frac{2}{1-v} s(x)-\frac{1}{1-v} T(v, x)+\frac{v}{1-v} s(x) L(v, x),
\end{aligned}
$$


defined for $(v, x) \in X:=(0,1) \times(-1,1]$, where we used the shorthand notations

$$
\begin{aligned}
s(x) & :=\sqrt{2-2 x} \\
T(v, x) & :=2 \sqrt{2+2 x} \arctan \frac{\sqrt{1-x^{2}}}{\frac{1+v}{1-v}+x}, \\
L(v, x) & :=\log \frac{\frac{1+v^{2}}{1-v^{2}}+x}{\frac{1+v^{2}}{1-v^{2}}-1} .
\end{aligned}
$$

The following estimate for $T(v, x)$ on $X$ will be useful later on:

$$
(1-v)(1+x) s(x)<T(v, x)<\frac{2(1+x) s(x)}{\frac{1+v}{1-v}+x} .
$$

The first inequality in (2.7) may be obtained by comparing the partial derivatives with respect to $v$ for fixed $x$, the second one readily follows from $\arctan y<y$ for $y>0$.

By the transformation

$$
v=v(\rho):=\left(\frac{1-\rho}{1+\rho}\right)^{2}, \quad x=x(\phi):=\cos 2 \phi,
$$

we define a bijective map $(\rho, \phi) \mapsto(v(\rho), x(\phi))$ of $(0,1) \times(0, \pi / 2]$ onto $X$. A straightforward calculation leads to the relations

$$
\begin{aligned}
& h(\rho, \phi)=(1+r)^{2} \cos \phi g(v(\rho), x(\phi)), \\
& p(\rho, \phi)=q(v(\rho), x(\phi)),
\end{aligned}
$$

between $h$ and $p$ and the new functions $g$ and $q$. We claim that the locus of the zeros of $g(v, x)$ is a curve $\gamma: t \mapsto(t, x(t)), t \in(0,1 / e]$, with

$$
\lim _{t \rightarrow 0} \gamma(t)=(0,1), \quad \lim _{t \rightarrow e^{-1}-} \gamma(t)=\left(e^{-1},-1\right),
$$

where $x^{\prime}(t)<0$ is continuous. The existence of such a curve $\gamma$ is guaranteed by the implicit function theorem since the partial derivatives

$$
\begin{aligned}
& g_{v}(v, x)=1+\frac{s(x)}{4(1+x)} T(v, x) \\
& g_{x}(v, x)=\frac{-1+v+x-v x+\frac{v}{2(1+x)} s(x) T(v, x)}{2\left(x^{2}-1\right)},
\end{aligned}
$$

of $g$ appear to be positive on $(0,1) \times(-1,1)$ by (2.7) and

$$
\lim _{v \rightarrow 0+} g(v, x)=\frac{1}{2} \log \frac{1+x}{2}<0, \quad \lim _{v \rightarrow 1-} g(v, x)=1,
$$

for fixed $x \in(-1,1)$. A computation of the limits

$$
\lim _{x \rightarrow 1-} g(v, x)=v, \quad \lim _{x \rightarrow-1+} g(v, x)=1+\log v
$$

for fixed $v$ proves the statement about the endpoints of $\gamma$.

We shall prove now that $q(v, x)$ is increasing on $\gamma$. To see this consider

$$
\frac{d}{d t} q(t, x(t))=q_{v}(t, x(t))-q_{x}(t, x(t)) \frac{g_{v}(t, x(t))}{g_{x}(t, x(t))}
$$


where

$$
\begin{aligned}
& q_{v}(v, x)=\frac{-T(v, x)+s(x)[2+L(v, x)]}{(1-v)^{2}}, \\
& q_{x}(v, x)=\frac{-T(v, x)-\frac{2 v(1+x)}{s(x)}[2+L(v, x)]}{2(1-v)(1+x)} .
\end{aligned}
$$

A straightforward computation involving (2.7) and $L(v, x) \geq 0$ shows $q_{v}(v, x)>0$ and $q_{x}(v, x)<0$ on $(0,1) \times(-1,1)$. Hence $\frac{d}{d t} q(t, x(t))>0$.

Translating our result via (2.8) and (2.9) back to the functions $h(\rho, \phi)$ and $p(\rho, \phi)$ we obtain the assertion.

We deduce from Lemma 2.5 that for $\lambda=i p$ and $p \geq 4 e /(e-1)$ the unique support point for $L_{\lambda}$ is the Koebe function $i K(-i z)$. If $p \leq-4 e /(e-1)$, then, by symmetry, the unique support point for $L_{\lambda}$ is $-i K(i z)$. This proves part (a) of Theorem 1.1.

To prove part (b), i.e., to show that for $0<p<4 e /(e-1)$, no rotation of the Koebe function is a support point for $L_{i p}$, we establish the following:

Lemma 2.6. For $0<p<4 e /(e-1)$ let $F_{0}$ be the uniquely determined $A(\rho, \phi(\rho))$ admissible function such that $p=p(\rho, \phi(\rho))$ for some $\rho \in((\sqrt{e}-1) /(\sqrt{e}+1), 1)$. Then $L_{i p}\left(F_{0}\right)>L_{i p}(i K(-i z))$.

Proof. We adopt the notation from the proof of Lemma 2.5. Using (2.2) we get $2 L_{i p}\left(F_{0}\right)=B_{0}-p \operatorname{Im} A_{2}$ with $B_{0}=2(\rho+1 / \rho+\cos 2 \phi) ; c f$. [SSp50], and, in view of (2.4) and the transformation (2.8),

$$
\operatorname{Im} A_{2}=\frac{T(v, x)-v L(v, x) s(x)}{2(1-v)}-s(x) .
$$

Thus we have to show that

$$
L_{i p}\left(F_{0}\right)-L_{i p}(i K(-i z))=2 \frac{1+v}{1-v}+x-\frac{q(v, x)}{2} \operatorname{Im} A_{2}-2 q(v, x)+3
$$

is non-negative for all $(v, x) \in X$ with $g(v, x)=0$. In fact, we will prove this for all $(v, x) \in X$. Let us denote the expression (2.12) by $R(v, x)$. Then we have

$$
\begin{aligned}
R(v, x)=\frac{1}{4(1-v)^{2}} & {\left[v^{2} s(x)^{2} L(v, x)^{2}-2 v s(x) T(v, x) L(v, x)+T(v, x)^{2}\right.} \\
& -2 s(x) v[4-4 v-2 s(x)+v s(x)] L(v, x) \\
& +2[4-4 v-2 s(x)+v s(x)] T(v, x) \\
& \left.+4(1-v)\left[5-v+x-4 s(x)+s(x)^{2}-v x\right]\right] .
\end{aligned}
$$

A straightforward calculation leads to

$$
4(1-v)^{2} R(v, x)=[T(v, x)-v s(x) L(v, x)]^{2}+2\left[c_{1}(v, x) a(v, x)+c_{0}(v, x)\right],
$$

where

$$
\begin{aligned}
a(v, x) & :=T(v, x)-v s(x) L(v, x), \\
c_{1}(v, x) & :=4(1-v)-(2-v) s(x), \\
c_{0}(v, x) & :=(1-v)[2-s(x)][2(3-v)-(1+v) s(x)] .
\end{aligned}
$$


Obviously, $c_{0}(v, x)>0$ for all $(v, x) \in X$. Furthermore, $L(v, x) \geq 0$ in $X$, since

$$
L_{v}(v, x)=-2 \frac{1+x}{v(1+x)+v^{3}(1-x)} \leq 0, \quad \lim _{v \rightarrow 1} L(v, x)=0 .
$$

Thus, the relations

$$
a_{v}(v, x)=-s(x) L(v, x), \quad \lim _{v \rightarrow 1} a(v, x)=0,
$$

show also $a(v, x) \geq 0$. In view of equation (2.13) it remains to prove that $c_{1}(v, x) a(v, x)+c_{0}(v, x) \geq 0$ for all $(v, x) \in X$ that satisfy $c_{1}(v, x)<0$, i.e. $v>2[2-s(x)] /[4-s(x)]$. We denote the set of all such points by $\Delta$ and show below that the partial derivative

$$
\begin{aligned}
\left(a+\frac{c_{0}}{c_{1}}\right)_{v}(v, x)= & -s(x) L(v, x)-\frac{4-s(x)^{2}}{4-s(x)} \\
& +\frac{s(x)[2-s(x)]\left[16-10 s(x)+3 s(x)^{2}\right]}{[4-s(x)][4-4 v-2 s(x)+v s(x)]^{2}}
\end{aligned}
$$

is positive for all $(v, x) \in \Delta$. Then the obvious limit relation

$$
\lim _{v \rightarrow 1}\left[a(v, x)+\frac{c_{0}(v, x)}{c_{1}(v, x)}\right]=0
$$

shows $a(v, x)+c_{0}(v, x) / c_{1}(v, x)$ is negative in $\Delta$. Thus $c_{1}(v, x) a(v, x)+c_{0}(v, x)$ is positive for all $(v, x) \in \Delta$.

To complete the proof we have to show that (2.14) is positive for all $(v, x) \in \Delta$. First, we use the estimates

$$
L(v, x)=\log \left(1+\frac{1+x}{\frac{1+v^{2}}{1-v^{2}}-1}\right) \leq \frac{1+x}{\frac{1+v^{2}}{1-v^{2}}-1}=-\frac{4-s(x)^{2}}{4}\left(1-\frac{1}{v^{2}}\right)
$$

and $16-10 s+3 s^{2} \geq 2(2+s)$ to obtain

$$
\begin{aligned}
\left(a+\frac{c_{0}}{c_{1}}\right)_{v}(v, x) \geq & -\frac{[2-s(x)]^{3}[2+s(x)]}{4[4-s(x)]}+s(x)\left[4-s(x)^{2}\right] \\
& \times\left(\frac{2}{[4-s(x)][4-4 v-2 s(x)+v s(x)]^{2}}-\frac{1}{4 v^{2}}\right) .
\end{aligned}
$$

Let us denote this lower bound by $P(v, x)$. The partial derivative of $P$ with respect to $v$ turns out to be

$$
P_{v}(v, x)=\frac{\left[4-s(x)^{2}\right] s(x)[s(x)-2](v-2)}{2 v^{3}[4-4 v-2 s(x)+v s(x)]^{3}} Q(v, s(x))
$$

where

$$
Q(v, s):=v^{2} s^{2}-10 v^{2} s-4 v s^{2}+28 v^{2}+28 v s+4 s^{2}-40 v-16 s+16 .
$$

In (2.15), the denominator is negative, since $(v, x) \in \Delta$, and the numerator is obviously non-negative. Since the critical values of $Q$ are $Q(0,2)=0$ and $Q(2,6)=48$, a limit argument shows that $Q(v, s) \geq 0$ for all $(v, s) \in \mathbb{R}^{2}$. Thus $P$ is monotonously decreasing as a function of $v$. Finally, using

$$
P(1, x)=\frac{[2-s(x)]^{2}[2+s(x)]}{s(x)[4-s(x)]}>0
$$

we conclude that $P(v, x)>0$ for all $(v, x) \in \Delta$. 


\section{REFERENCES}

[Bom67] E. Bombieri, On the local maximum property of the Koebe function, Inv. Math. 4 (1967), 26-67. MR 36:1635

[BH85] D. Bshouty, W. Hengartner, Local behavior of coefficients in subclasses of $\mathcal{S}$, Contemp. Math. 38 (1985), 77-84. MR 86m:30007

[BH87] D. Bshouty, W. Hengartner, A variation of the Koebe mapping in a dense subset of $\mathcal{S}$, Canad. J. Math. 39 (1987), no. 7, 54-73. MR 88j:30048.

[Bro81] J. E. Brown, Univalent functions maximizing $\operatorname{Re}\left(a_{3}+\lambda a_{2}\right)$, Ill. J. Math. 25, No. 3 (1981), 446-454. MR 82j:30022

[Dur83] P. L. Duren, Univalent Functions, Springer-Verlag, 1983. MR 85j:30034

[Haa83] H. Haario, On the extreme points of classes of univalent functions, Ann. Acad. Sci. Fenn., Ser. A. I. Math. 8 (1983), 55-66. MR 84d:30019

[KT80] R. A. Kortram, O. Tammi, Non-homogeneous combinations of coefficients of univalent functions, Ann. Acad. Sci. Fenn., Ser. A. I. Math. 5 (1980), 131-144. MR 81m:30017

[Leu85] Y. I. Leung, Notes on Loewner differential equations, Contemp. Math. 38 (1985), 1-11. MR 86m:30007

[Pfl88] A. Pfluger, On the functional $a_{3}-\lambda a_{2}^{2}$ in the class $\mathcal{S}$, Comp. Var. 10 (1988), 83-95. MR 89e:30034

[SSp50] A. C. Schaeffer, D. C. Spencer, Coefficient Regions for Schlicht Functions, AMS Coll. Series, 1950. MR 12:326c

[Tam82] O. Tammi, Extremum Problems for Bounded Univalent Functions, II, Springer LNM 913, 1982. MR 84a:30032

Department of Mathematics, Bayerische Julius-Maximilians-Universität, Am HubLAND, D-97074 WÜrzBurg, Germany

E-mail address: greiner@mathematik.uni-wuerzburg.de

Department of Mathematics, Bayerische Julius-Maximilians-Universität, Am HubLAND, D-97074 Würzburg, Germany

Current address: Department of Mathematics, University of Michigan, Ann Arbor, Michigan 48109

E-mail address: roth@mathematik.uni-wuerzburg.de 